\title{
Making a diagnosis of androgen deficiency in adult men: what to do until all the facts are in?
}

\author{
Shalender Bhasin and Frederick Wu
}

We find it ironic that-4 decades after the introduction of testosterone immunoassays - we should be writing this lament on the state of the art of diagnosing androgen deficiency in men. The Endocrine Society Clinical Practice Guideline on testosterone therapy emphasized that androgen deficiency is a clinical syndrome which should be diagnosed only in men with characteristic symptoms and signs, and unequivocally low testosterone levels (Bhasin S et al. [2006] J Clin Endocrinol Metab 19: 1995-2010). It remains, however, that the clinical features of androgen deficiency are often nonspecific and based largely on empirical experience in severely androgen-deficient young men, in whom recognizable additional features of underlying etiologies (e.g. small testes in men with Klinefelter's syndrome or anosmia in men with idiopathic hypogonadotropic hypogonadism) help corroborate the diagnosis. This well-practiced diagnostic approach is frequently found wanting when dealing with milder forms of androgen deficiency, especially in older men with an age-related decline in testosterone levels on a background of non-hormone-related complaints. Furthermore, we do not know the threshold level(s) of testosterone below which consequences of androgen deficiency become manifest, and it remains unclear which and how many clinical symptoms can best establish the diagnosis of androgen deficiency.

Reference limits for testosterone levels generated in a population-based, random sample with a reliable method and a standardized calibrator are not available but are badly needed. Consequently, partitioning of testosterone levels into 'normal' or 'low' categories is fraught with a substantial risk of misclassification.

Additionally, there is growing concern about the accuracy of many commercial testosterone immunoassays, especially in the low concentration range. Liquid chromatography-tandem mass spectrometry (LC-MS/MS) provides accurate and precise measurements of testosterone concentrations, even in the low range; however, the LCMS/MS assay is expensive and experience of its use is limited. The normative ranges also
It remains

unclear which

and how many

symptoms can

best establish

the diagnosis

of androgen

deficiency

$S$ Bhasin is Professor of Medicine at Boston University School of Medicine, Boston, MA, USA and FWu is Professor of Medicine and Endocrinology, University of Manchester, Manchester, UK.

Competing interests

$S$ Bhasin has declared associations with the

following companies:

Auxilium Pharmaceuticals and Solvay Pharmaceuticals. See the article online for full details of the relationship.

F Wu declared he has no

competing interests.

www.nature.com/clinicalpractice doi:10.1038/ncpendmet0299 vary among different assays. The assays for the measurement of free (i.e. not bound to albumin or sex-hormone-binding globulin) or bioavailable testosterone are even more problematic; therefore, total, rather than free, testosterone levels should be used initially to confirm the clinical diagnosis.

Epidemiologic data on the association of various testosterone thresholds with outcomes should be used to establish numerical boundaries for testosterone levels. Diagnostic algorithms based on a combination of validated signs, symptoms, and population-based reference limits, and investigated in randomized controlled trials, would provide a rational basis for partitioning men into androgen-deficient and androgen-replete categories.

What should a practicing physician do until such validated algorithms become available? First, testosterone levels should be measured only in men with symptoms and signs of androgen deficiency, as suggested by The Endocrine Society guidelines. Second, rather than with unvalidated screening questionnaires, clinical features should be critically assessed in individual patients with the awareness that sexual and physical symptoms, especially in combination, are more informative than psychological or behavioral ones, as suggested by the European Male Ageing Study (Arnott JM et al. [2006] A clinically relevant approach to defining the prevalence of late onset hypogonadism in men from the general population. Abstract \#OR5-4 presented at The Endocrine Society's 88 ${ }^{\text {th }}$ Annual Meeting: 2006 24-27 June, Boston, MA). Third, measurement of total testosterone levels by a reliable assay, such as LCMS/MS, preferably in the morning, should be the initial diagnostic test. Fourth, physicians should use the normative ranges specific to that assay. Fifth, because a substantial proportion of men with an initial testosterone level below the lower limit will have a repeat testosterone level within the normal range, a low testosterone level should be confirmed by repeat measurement. These steps should help reduce the risk of misclassifying androgen deficiency in men. 